\title{
Lithium-Rich K Giants with Infrared Excesses: Fundamental Parameters and CNO Abundances
}

\author{
Natalia A. Drake ${ }^{1,2}$, Ramiro de la Reza ${ }^{1}$ and Licio da Silva ${ }^{1}$ \\ ${ }^{1}$ Observatório Nacional/MCT, R. José Cristino, 77 CEP:20921-400, \\ Rio de Janeiro, Brazil \\ 2 Astronomical Institute of St. Petersburg University, St. Petersburg, \\ 198904 Russia
}

\begin{abstract}
We present the results of an analysis of seven faint $\mathrm{K}$ giants with strong Li lines, recently discovered during the Picodos Dias urvey, from high-resolution and high signal-to-noise-ratio spectra. The fundamental parameters of these stars, such as effective temperature, surface gravity, metallicity and microturbulence, were determined. We also obtained LTE abundances of $\mathrm{Li}$ and $\mathrm{CNO}$ elements and measured the ${ }^{12} \mathrm{C} /{ }^{13} \mathrm{C}$ ratios by spectral synthesis using the lines of $\mathrm{Li}$ I ( $\lambda 6104 \AA$ and $\lambda 6708 \AA$ ), $\mathrm{C}_{2}, \mathrm{CN}$, and [O I]. The studied Li K stars are found to be red giant branch stars with masses around $1 M_{\odot}$.
\end{abstract}

\section{Introduction}

A small part of the red giant stars $(\sim 1 \%)$ have been found to have high lithium abundances and the great majority of these Li-rich $\mathrm{K}$ giants have IR excesses which can be caused by an existence of circumstellar shells. de la Reza, Drake, \& da Silva (1996) and de la Reza et al. (1997) suggested that Li-rich giants are not peculiar stars and that all low mass $\left(\$ 2.5 M_{\odot}\right)$ stars pass one or several times through a short Li-rich phase during the RGB stage. A Li enrichment mechanism acting in low mass red giants - cool bottom processing - was proposed by Sackmann \& Boothroyd (1999). More discussion on this subject can be found in Sackmann \& Boothroyd in these proceedings. In order to understand the evolution stage when $\mathrm{Li}$ enrichment and shell formation occur, we carried out a detailed spectral analysis of seven red giants with strong Li lines discovered during the Pico dos Dias Survey (PDS) (see Torres et al. in this Proceedings).

\section{Observations}

The high-dispersion spectrograms used in this analysis were obtained in Chile with the telescopes of $3.60 \mathrm{~m}$ at La Silla, ESO, (PDS 3=HD 19745, PDS 68, PDS 432) and of $4.0 \mathrm{~m}$ at Tololo, CTIO, (PDS 132, PDS 354, PDS 365, PDS 524). Standard NOAO's IRAF procedures were used for the data reduction. 


\section{Fundamental Stellar Parameters, Li Abundances and ${ }^{12} \mathrm{C} /{ }^{13} \mathrm{C}$}

Using an iterative procedure we determined the effective temperatures and microturbulence velocities by obtaining iron abundances independent of excitation potentials and measured equivalent widths respectively. The surface gravities were derived from the ionization equilibrium. The photospheric models of $\mathrm{Ku}-$ rucz (1993) and Bell et al. (1976) were used. Because the faint giants studied here have no measured parallax, we adopt absolute magnitude values corresponding to their spectral and luminosity classes. Using obtained values of the temperature, superficial gravity and luminosity and photometric data we were able to estimate the stellar masses. To derive $\mathrm{CNO}$ and $\mathrm{Li}$ abundances and the ${ }^{12} \mathrm{C} /{ }^{13} \mathrm{C}$ isotopic ratios we realized a detailed LTE spectral synthesis analysis of each star in the spectral regions containing the lines of $\mathrm{C}_{2},{ }^{12} \mathrm{CN},{ }^{13} \mathrm{CN},[\mathrm{OI}]$ and $\mathrm{LiI}(\lambda 6104 \AA$ and $\lambda 6708 \AA)$ using the last version of the MOOG program. The molecular line lists and the analysis details can be found in Drake (1998). The determined fundamental parameters, Li abundances and ${ }^{12} \mathrm{C} /{ }^{13} \mathrm{C}$ ratios are presented in the table. The values of ${ }^{12} \mathrm{C} /{ }^{13} \mathrm{C}$ are in the limits from 9 till 35 and are typical of the red giant branch stars. Comparing measured ${ }^{12} \mathrm{C} /{ }^{13} \mathrm{C}$ ratios and $\mathrm{Li}$ abundances we derived that the enrichment of ${ }^{13} \mathrm{C}$ relative to ${ }^{12} \mathrm{C}$ on the surfaces of Li-rich $\mathrm{K}$ giants is not correlated with that of ${ }^{7} \mathrm{Li}$.

Table 1. Associated IRAS sources and determined stellar parameters

\begin{tabular}{lccccccc}
\hline Star & IRAS & $\begin{array}{c}T_{\text {eff }} \\
\mathrm{K}\end{array}$ & $\log g$ & {$[\mathrm{Fe} / \mathrm{H}]$} & $\begin{array}{c}M \\
M_{\odot}\end{array}$ & ${ }^{12} \mathrm{C} /{ }^{13} \mathrm{C}$ & $\begin{array}{c}\log \epsilon(\mathrm{Li}) \\
\lambda 6708 / \lambda 6104\end{array}$ \\
\hline HD 19745 & $03062-6538$ & 4700 & 2.3 & 0.08 & 1.2 & 30 & $3.9 / 3.9$ \\
PDS 68 & $13539-4153$ & 4140 & 1.4 & -0.40 & 1.2 & 20 & $3.3 / 3.9$ \\
PDS 132 & $07227-1320$ & 3910 & 1.7 & -0.01 & 2.0 & 12 & $0.8 / \leq 1.5$ \\
PDS 354 & $12236-6302$ & 4710 & 2.4 & 0.22 & 1.2 & 9 & $1.7 / 2.8$ \\
PDS 365 & $13313-5838$ & 4540 & 2.2 & -0.09 & 1.1 & 12 & $3.3 / 3.3$ \\
PDS 432 & $16514-4625$ & 4580 & 2.0 & 0.28 & 0.7 & 35 & $2.9 / 3.4$ \\
PDS 524 & $18334-0631$ & 4160 & 0.8 & 0.00 & 1.0 & 20 & $0.7 / \leq 1.4$ \\
\hline \hline
\end{tabular}

Acknowledgments. N.A.D. thanks FAPERJ for the financial support under the contract E-26/151.172/98 and R. dela R. and L. daS. thank CNPq for the grants $200580 / 97-3$ and $301375 / 86-0$ respectively.

\section{References}

Bell, R.A., Eriksson, K., Gustafsson, B., \& Nordlund, A. 1976, A\&AS, 23, 37 de la Reza, R., Drake, N.A., da Silva, L. 1996, ApJ, 456, L115

de la Reza, R., Drake, N.A., da Silva, L., Torres, C.A.O., \& Martin, E.L. 1997, ApJ, 482, L77

Drake, N.A. 1998, PhD Thesis, Observatório Nacional, Rio de Janeiro Kurucz, R.L. 1993, ATLAS9, Smithsonian Astroph. Obs. CD-ROM no. 13 Sackmann, I.-J., \& Boothroyd, A.I. 1999, ApJ, 510, 217 